\title{
High-Frequency Vibrational Properties of Metallic Nanocrystalline Grain Boundaries
}

\author{
P. M. Derlet and H. Van Swygenhoven \\ Paul Scherrer Institute, 5232 Villigen, Switzerland \\ (Received 26 May 2003; published 23 January 2004)
}

\begin{abstract}
The high-frequency phonon properties of a computer generated nanocrystalline (nc) fcc Ni with a mean grain size of $5 \mathrm{~nm}$ are investigated by directly calculating the on-site phonon Green's function using a recursion technique based on a continued fraction representation. It is found that the highfrequency tail, observed in both experiment and previous simulation work, arises primarily from spatially confined vibrational modes forming within the nc grain boundary regions.
\end{abstract}

PACS numbers: 81.07.Bc, 02.70.Ns, 63.22.+m

It is known that the large amount of grain boundaries (GBs) in nanocrystalline (nc) materials can influence to a great extent thermodynamic properties such as heat capacity and thermal expansion [1-3]. Moreover, it has been recently demonstrated that these thermodynamic properties do not only depend on the grain size, but they are also very dependent on the microstrain (controlled by annealing procedures), suggesting the importance of GB structures [4]. Central to these quantities is the distribution and nature of nc phonon modes where, via thermal neutron scattering experiments [5-9], it has been established that the nc vibrational density of states (VDOS) contains both a high- and a low-frequency enhancement. Previous simulation work by us [10] established that both the low- and high-frequency enhancement seen in the experimental VDOS can be directly attributed to the VDOS arising from the atoms in the GB region, while the VDOS within grains differs little from the corresponding fcc crystalline VDOS. In addition, a low-frequency nonquadratic power law was found for the GB component suggesting a reduced dimensionality effect.

Until now the VDOS for systems containing the large number of atoms necessary to capture a realistic nc GB structure has been mainly obtained from the Fourier transform of the velocity-velocity autocorrelation function derived from a finite temperature molecular dynamics simulation $[10,11]$. Although reasonably efficient in calculating the VDOS averaged over different atomic environments, it lacks the computational efficiency and phonon energy resolution to investigate the fine detail with respect to local environment. This is particularly prevalent for the high-frequency regime, which is overly sensitive to the nearest neighbor atomic scale.

In the present Letter we investigate for the first time the origin of the high-frequency tail inherent to the nc-VDOS using phonon Green's function (GF) recursion techniques to calculate the VDOS of particular atoms within the nc GB. We find that the origin of the high-frequency tail lies in spatially confined states within the GB forming above the bulk band of propagating phonon modes, their spatial location corresponding to regions of reduced coordination within the GB region.
The VDOS is calculated directly from the imaginary part of the on-site phonon GF, which can be derived formally from the general intersite phonon GF:

$$
G_{i j}^{\mu \nu}(\omega)=\left[\left(\tilde{I} \omega^{2}-\tilde{\Delta}\right)^{-1}\right]_{\mu \nu, i j},
$$

where $\tilde{\Delta}$ is the Hessian matrix (of $\operatorname{rank} 3 N$ ) of an $N$ atom system. Here the atomic site indices $i$ and $j$ span from 1 to $N$, and the polarization directions $\mu$ and $\nu$ from 1 to 3 . To obtain the full Green's function via Eq. (1) a matrix inversion is required, which becomes an intractable problem for systems containing a large number of atoms. Hence we employ an $O(N)$ recursion technique to calculate efficiently the on-site GF for particular atoms of interest within a given atomic nc configuration. Similar techniques have most recently been used for the efficient calculation of electronic GFs in the development of analytical bond-order potentials $[12,13]$.

In the numerical scheme, the on-site GF is represented as a finite continued fraction,

$$
\left[G_{i i}^{\mu \mu}(\omega)\right]^{-1}=\omega^{2}-a_{0}-b_{1}^{2} /\left[\omega^{2}-a_{1}-b_{2}^{2} /(\ldots)\right],
$$

where $a_{n}$ and $b_{n}$ are the recursion coefficients that can be associated with the moments of the corresponding VDOS. In practice this first involves tridiagonalizing the Hessian via a Lanczos algorithm [14], where the starting Lanczos state is set equal to the atom of interest. In its tridiagonal form, the diagonal and off-diagonal elements of the Hessian are simply equal to $a_{n}$ and $b_{n}$, respectively. Convergence of the recursion coefficients with respect to $n$ allows the continued fraction to be terminated (via a square root terminator function [12] with an appropriate choice of the terminating recursion coefficients, $\tilde{a}_{\infty}$ and $\tilde{b}_{\infty}$ ) at a level far less than $3 N$, since the higher order recursion coefficients represent less local and more distant structural information not necessary to the evaluation of the on-site GF. Such a mathematical representation of the GF is found to be well suited to numerical evaluation. Convergence can be greatly improved by exploiting a generalization of the Lanczos procedure that block tridiagonalizes the Hessian [13,15]. Presently this results in an $N \times N$ tridiagonal Hessian where each 
recursion coefficient is now a $3 \times 3$ matrix containing the original symmetry of the on-site polarization tensor. Equation (2) must then also be generalized to a continued fraction matrix expansion where, for termination, a diagonal square root terminator matrix is now employed [15].

The "model" nc-Ni atomic configuration used in the present work is part of a family of samples used extensively in past work studying nc structure and mechanical properties $[16,17]$. It contains a fully three-dimensional GB network based on the Voronoi construction [18]. Since recent work has established that the shape of the normalized GB VDOS is largely independent of grain size [10], we choose for this study a sample containing 15 grains at a mean grain size of $5.2 \mathrm{~nm}$, totalling $\sim 120000$ atoms. The configuration is derived from a molecular dynamics simulation at $300 \mathrm{~K}$ within the Parrinello-Rahman framework [19], in which all atoms and the simulation cell side lengths are averaged over 1 ps using the second moment tight binding potential of Cleri and Rosato [20], which has been used extensively for the investigation of defect vibrational properties in Ni $[21,22]$. From this, the simulation cell side lengths are held fixed, and all atomic coordinates are further relaxed to their local minima using molecular statics. The Hessian itself is determined directly from the second derivative with respect to atomic coordinates of the system's total energy. Thus the procedure represents a quasiharmonic calculation with respect to thermal expansion at room temperature [23].

Figure 1(a) displays the average VDOS for the GB and the grain interior calculated using block recursion coefficients up to 60 levels. In both cases, the average was derived from 1000 arbitrarily chosen atoms with a local crystallinity class of non-fcc and fcc atoms, respectively, determined via the common nearest neighbor analysis of Honeycutt and Andersen [24] (see also Ref. [25]). For comparison the VDOS for a perfect fcc crystalline $\mathrm{Ni}$ sample, containing approximately the same number of atoms as the entire nc sample, is also shown (comparing well to the experimentally derived fcc Ni VDOS [26]). In
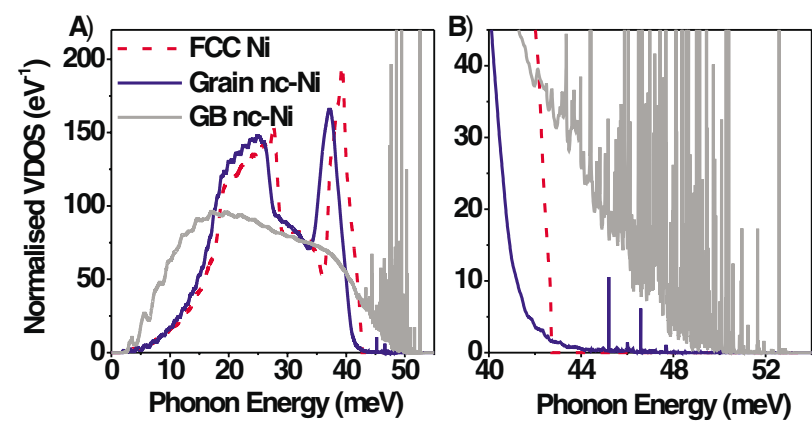

FIG. 1 (color online). (a) Normalized VDOS for crystalline fcc Ni, and the average VDOS's for the grain and GB components of nc-Ni with an average grain diameter of $5.2 \mathrm{~nm}$. (b) A blowup of the high-frequency region. this case only 30 block recursion levels were needed for general convergence. The nc-Ni GB VDOS displays a nonquadratic low-energy power law and a significant high energy tail, whereas the grain interior VDOS, apart from a small shift and some broadening (particularly in the region of the longitudinal Van Hove peak), does not differ significantly from the fcc VDOS. These features reconfirm our earlier VDOS results derived from finite temperature velocity-velocity autocorrelation methods [10] that the anomalous nc VDOS can be directly attributed to the vibrational properties of the nc GB atoms. The total nc VDOS (not shown) arising from the grain and GB contributions compares well to the experimental nc-Ni VDOS [7], where the high-frequency enhancement of the GB component results in an effective broadening of the observed longitudinal Van Hove peak.

Figure 1(b) is a blowup of the high-frequency region for the three VDOS's considered in Fig. 1(a). For the GB VDOS, the degree of noise greatly increases beyond the band of propagating modes (defined approximately by the fcc crystalline VDOS). Since the GB VDOS is derived from the individual VDOSs of $1000 \mathrm{~GB}$ atoms, this indicates that the high-frequency VDOS varies significantly as a function of GB environment. The VDOSs of individual GB atoms reflect this by being quite different from each other in the high-frequency regime, containing a number of narrow singular peaks at a density less than that seen in the average GB VDOS. This result demonstrates the main advantage of the present technique, since such detail is largely smeared out using velocity-velocity correlation methods due to the anharmonicities present in the finite temperature molecular dynamics. Moreover, due to the quasiharmonic nature of the calculation, Fig. 1 establishes without doubt that the high-frequency enhancement arises fundamentally from the structural properties of the GB system.

The use of a square-root terminator brings with it the possibility of spurious results that can depend sensitively on the choice of the termination recursion matrix coefficients, $\tilde{a}_{\infty}$ and $\tilde{b}_{\infty}$. Figure 2 displays the VDOS for a single GB atom for a narrow range of high-frequency energies, for six recursion termination levels. For each

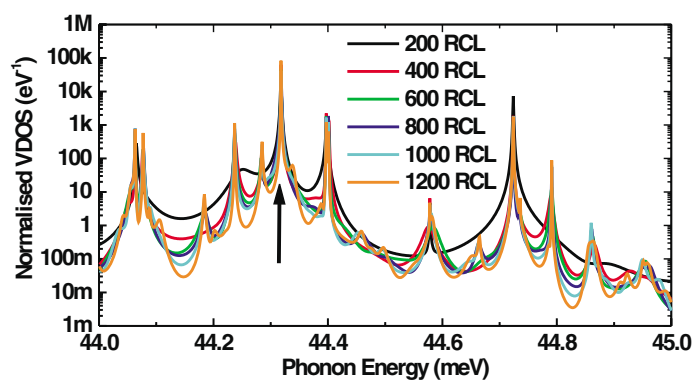

FIG. 2 (color online). VDOS for one GB atom for a narrow range of energies for different recursion termination levels. 
termination level, the termination recursion matrix coefficients, $\tilde{a}_{\infty}$ and $\tilde{b}_{\infty}$, were taken as the recursion matrix coefficients at that particular termination level. The figure demonstrates that with an increasing termination level, the high-frequency singular structure becomes consistently more resolved. Indeed convergence in terms of peak area-the effective contribution of the associated eigenmode to the VDOS - is relatively quick occurring between 100 and 200 recursion levels. We note also that the displayed modes also are reproduced using a zero terminator (that is, using a finite number of recursion levels without a terminator function) in conjunction with a phonon energy containing a small imaginary component. Such a result suggests that the singular structure does indeed correspond to the actual high-frequency eigenvalue spectrum of the Hessian and is not an artifact of the numerical technique. Moreover, such modes are sensitive only to the first 200 block recursion levels, indicating a dependence primarily on the local environment around the atomic site of interest.

To investigate the structural origin of the singular high-frequency regime we now consider the VDOS that arises from a particular GB. Figure 3(a) is an atomic section of a GB with the viewing direction along the GB plane. Here atoms are marked according to their local crystallinity class [24], where grey atoms represent fcc and non-grey other 12 coordinated and non-12 coordinated symmetries. Thus the nongrey atoms represent the GB interface region. This GB may be considered a general high-angle interface and has been analyzed in detail in previous work [25] in which (111) planes of the upper grain and the (100) planes of the lower grain meet to form regions of misfit. Figures 3(b)-3(d) display only the nonfcc atoms of Fig. 3(a) with the viewing direction now along the GB plane normal, where the atoms are

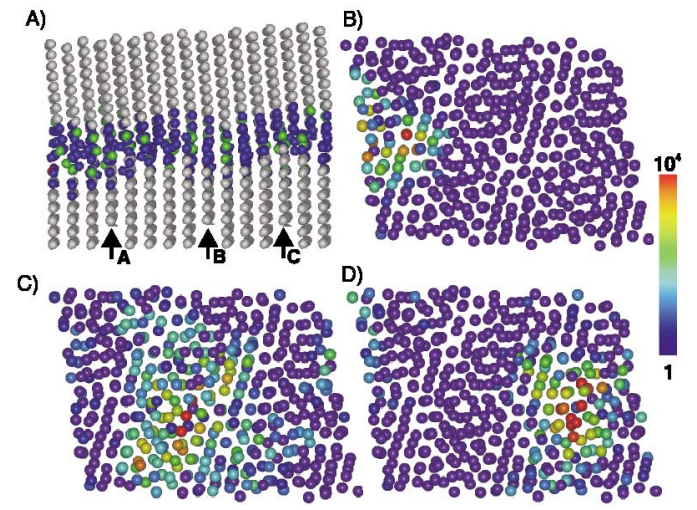

FIG. 3 (color online). (a) A high-angle GB with viewing direction along the GB plane, in which the atoms are marked according to their local crystalline symmetry. (b)-(d) are views of the non-fcc atoms with viewing direction along the normal of the GB plane in which the atoms are marked according to their VDOS oscillator strength for three respective phonon frequencies. now marked according to their VDOS value for three high-frequency phonon energies: 46.024, 44.318, and $44.791 \mathrm{meV}$. The marking scheme is logarithmic following the scale indicated in the figure.

Inspection of Figs. 3(b) -3(d) reveals that such singular VDOS structures correspond to high-frequency oscillation modes that are spatially confined typically over a number of atoms. Such behavior exists throughout the GB network at a variety of high-frequency phonon energies and demonstrates that the high-frequency enhancement in the GB VDOS originates from spatially confined modes forming within the GB. That at least 200 recursion levels are needed for good convergence indicates that these modes may extend in a nontrivial way over a number of atoms. We note that Fig. 2 details the VDOS for that atom having the highest oscillator strength in Fig. 3(c), the arrow in Fig. 2 indicating the corresponding peak at $44.318 \mathrm{meV}$.

Figures 4(a)-4(c) now show a view perpendicular to those planes [indicated by the arrows in Fig. 3(a)] where spatially confined modes are observed. Again, the GB atoms are marked according to their VDOS strength, and the smaller fcc atoms of the grains are shaded grey. The nearest neighbor coordination of some of the GB atoms is also displayed. Note that Fig. 4(c) occurs at a region of misfit so two upper 111 planes were included in the plot. The spatially confined modes are again evident and from evaluation of the VDOS of the nearby fcc atoms, such modes are found to extend sometimes up to one or two nearest neighbor distances into the grains. For the three phonon energies studied, it was observed that the atom containing the highest VDOS value (indicated by arrows in Fig. 4) has locally the lowest, or one of the lowest coordinations. Such a reduction in coordination need not necessarily be related to free volume within the GB and can be simply a result of the misorientation at the interface between corresponding grains. Nevertheless,

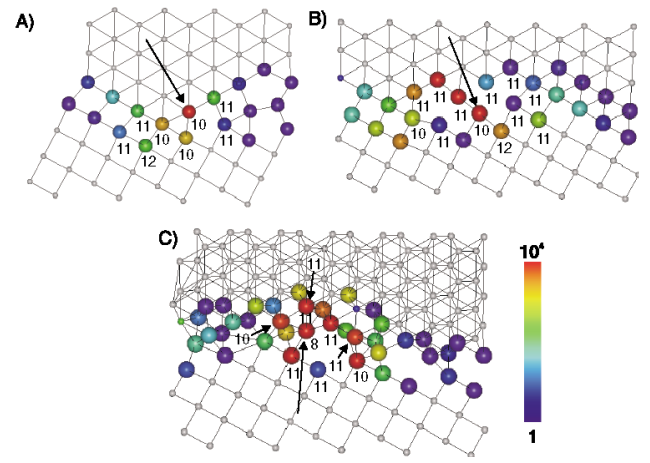

FIG. 4 (color online). (a) Atomic 111/100 plane of upper/ lower grain indicated by arrow A in Fig. 3(a). (b) Atomic $111 / 100$ plane indicated by arrow B and (c) atomic 111/100 plane indicated by arrow $\mathrm{C}$. GB atoms are marked according to their VDOS oscillator strength and the smaller grey circles represent fcc atoms. 
Fig. 4(c) displays a particular spatially confined mode spanning several atoms all of which surround an area of free volume identified by theoretical positron lifetime calculations [27], which is the size of a GB vacancy.

Such vibrational modes were also seen in other GBs containing more clearly defined regions of order and disorder than that seen in Fig. 3(a). For example, spatially confined modes were observed in the cores of well separated GB dislocations and were also seen at the disordered step regions between twin boundary structural units in a GB close to a $\Sigma=3$ orientation. In both cases, these regions again could be associated with regions of reduced coordination. The corresponding VDOS of these GBs exhibit a reduced high-frequency tail component when compared to the VDOS of the GB shown in Figs. 3(a), due to the reduced number of atoms that are non-12 coordinated. In terms of the average nc-VDOS, the degree to which GBs of a particular misorientation contribute to the high-frequency tail is thus expected to be a sensitive function of the resulting atomic scale interface structure.

The present approach reveals qualitatively some degree of spatial confinement; however, it is only through the intersite GF that a quantitative characterization of the degree of localization can be made. Nevertheless, such an observation suggests that the high-frequency spatially confined modes arise from a reduction in nearest neighbor coordination within the GB regions. From the unsaturated nature of the metallic bond, a reduction in coordination leads to a strengthening of the remaining bonds [28] and a corresponding stiffening in the force matrix $\tilde{\Delta}$ used in Eq. (1). Inspection of the force matrix for the GB atoms indicates that the largest effect occurs in the block diagonals for those atoms with reduced coordination. Such a local stiffening of the diagonal terms of the force matrix is known to lead to the formation of localized oscillation modes [29] and is the more likely origin of the high-frequency tail seen in Fig. 1 than say the vibrational analog of Anderson localization [30], since the nc GBs are in general not so strongly disordered containing only minor positional disorder (atoms that cannot be associated with a lattice position of any neighboring grain) [25].

In conclusion, we have investigated the high-frequency regime of the vibrational density of states for nanocrystalline fcc metallic systems using phonon Green's function methods and have established that the highfrequency tail inherent to the nc-VDOS arises primarily from spatially confined states forming within the GB region above the main band of propagating modes. Such modes occur within the GB at atoms having a reduced coordination that can often be associated with free volume within the GB region.

The authors thank David Pettifor and Sergei Dudarev for helpful discussions.
[1] J. Rupp and R. Birringer, Phys. Rev. B 36, 7888 (1987).

[2] Y.Y. Chen, Y. D. Yao, S.S. Hsiao, S. U. Jen, B.T. Lin, H. M. Lin, and C. Y. Tung, Phys. Rev. B 52, 9364 (1995).

[3] N. X. Sun and K. Lu, Phys. Rev. B 54, 6058 (1996).

[4] L. H. Qian, S. C. Wang, Y. H. Zhao, and K. Lu, Acta Mater. 50, 3425 (2002).

[5] B. Fultz, J. L. Robertson, T. A. Stephens, L. J. Nagel, and S. Spooner, J. Appl. Phys. 79, 8318 (1996).

[6] H. N. Frase, L. J. Nagel, J. L. Robertson, and B. Fultz, Philos. Mag. B 75, 335 (1997).

[7] E. Bonetti, L. Pasquini, E. Sampaolesi, A. Deriu, and G. Cicognani, J. Appl. Phys. 88, 4571 (2000).

[8] U. Stuhr, H. Wipf, K. H. Andersen, and H. Hahn, Phys. Rev. Lett. 81, 1449 (1998).

[9] L. Pasquini, A. Barla, A. I. Chumakov, O. Leupold, R. Rüffer, A. Deriu, and E. Bonetti, Phys. Rev. B 66, 073410 (2002).

[10] P. M. Derlet, R. Meyer, L. J. Lewis, U. Stuhr, and H. Van Swygenhoven, Phys. Rev. Lett. 87, 205501 (2001).

[11] D. Wolf, J. Wang, S. R. Phillpot, and H. Gleiter, Phys. Rev. Lett. 74, 4686 (1995).

[12] A. P. Horsfield, A. M. Bratovsky, M. Fearn, D. G. Pettifor, and M. Aoki, Phys. Rev. B 53, 12694 (1996).

[13] T. Ozaki, M. Aoki, and D. G. Pettifor, Phys. Rev. B 61, 7972 (2000).

[14] C. Lanczos, J. Res. Natl. Bur. Stand. 45, 255 (1950).

[15] J. Inoue and Y. Ohta, J. Phys. C 20, 1947 (1987).

[16] H. Van Swygenhoven, Science 296, 66 (2002).

[17] P. M. Derlet, A. Hasnaoui, and H. Van Swygenhoven, Scr. Mater. 49, 629 (2003).

[18] G. Z. Voronoi, J. Reine Angew. Math. 134, 199 (1908).

[19] M. Parrinello and A. Rahman, J. Appl. Phys. 52, 7182 (1981).

[20] F. Cleri and V. Rosato, Phys. Rev. B 48, 22 (1993).

[21] R. Meyer and L. J. Lewis, Phys. Rev. B 66, 052106 (2002).

[22] R. Meyer, L. J. Lewis, S. Prakash, and P. Entel, Phys. Rev. B 68, 104303 (2003).

[23] N.W. Ashcroft and N. D. Mermin, Solid State Physics (Saunders College Publishing, Philadelphia, 1988), Chap. 25.

[24] J. D. Honeycutt and H. C. Andersen, J. Phys. Chem. 91, 4950 (1987).

[25] P. M. Derlet and H. Van Swygenhoven, Phys. Rev. B 67, 014202 (2002).

[26] Metals: Phonon States, Electronic States and Fermi Surfaces, Landolt-Börnstein Vol. 13 (Springer-Verlag, Berlin, Heidelberg, New York, 1981), p. 104.

[27] S. Van Petegem, Doctoral thesis, University of Ghent, 2003.

[28] D. Pettifor, Bonding and Structure of Molecules and Solids (Clarendon Press, Oxford, 1995), Chap. 5.

[29] W. A. Harrison, Solid State Theory (Dover Publications, New York, 1979), Chap. 4.

[30] J. J. Ludlam, S. N. Taraskin, and S. R. Elliott, Phys. Rev. B 67, 132203 (2003). 